\title{
Selectivity of the plant growth regulators trinexapac-ethyl and sulfometuron-methyl to cultivated species
}

\author{
Núbia Maria Correia*, Gilson José Leite
}

UNESP/FCAV - Depto. de Fitossanidade, Via de Acesso Prof. Paulo Donato Castellane, s/n. - 14884-900 - Jaboticabal, $\mathrm{SP}$ - Brasil.

${ }^{*}$ Corresponding author <correianm@fcav.unesp.br>

Edited by: Leonardo Oliveira Medici

\begin{abstract}
The aerial spraying of plant ripeners on sugar cane (Saccharum officinarum L.) crops causes often the contamination of neighboring areas, which subsidizes formal complaints from the neighbors. These contaminations are due to spraying taking place during inadequate environmental conditions or from technical mistakes during the application. One of the most important causes of this contamination is the susceptibility of the species being cultivated surrounding sugar cane. In order to evaluate the effects of sugar cane plant ripeners trinexapac-ethyl and sulfometuronmethyl on peanuts, cotton, potato, coffee, citrus, beans, sunflower, cassava, rubber, soybean, and grapes, eleven experiments - one for each species - were carried out from May 2009 to Jan. 2010. The field experiment was set according to a completely random design with five treatments and four replications. Just before or during flowering, a single treatment of trinexapac-ethyl at 100

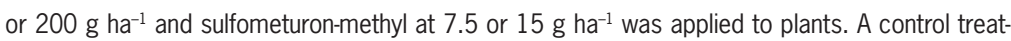
ment (plants not treated) for each species was part of each experiment. Trinexapac, at the doses of 100 and $200 \mathrm{~g} \mathrm{ha}^{-1}$, showed selectivity to peanuts, cotton, potato, coffee, citrus, sunflower, cassava, rubber, soybean, and grape. At the lowest dose (100 $\left.\mathrm{g} \mathrm{ha}^{-1}\right)$, it was selective for bean. Sulfometuron, at the dose of $7.5 \mathrm{~g} \mathrm{ha}^{-1}$, was selective for peanuts and, at the two studied doses (7.5 and $15 \mathrm{~g} \mathrm{ha}^{-1}$ ), it was selective for coffee, citrus, cassava, and rubber.

Keywords: phytointoxication, ripener, sugar cane
\end{abstract}

Received February 10, 2011

Accepted December 01, 2011 cochemical properties, toxicological proofs, residual analysis in foods, efficacy certificate, and selectivity to the target crop. But, tests to essay the response of non-target crops to herbicides are not demanded since the drift risk or application mistakes are unpredictable field events. This evaluation should nonetheless be part of the requirements, at least for herbicides registered to be aerially sprayed due to the increased possibility of contamination.

Trinexapac-ethyl and sulfometuron-methyl are the most widely used sugar cane plants ripeners. Thus, the determination of their degree of toxicity to important crops is necessary. This study aimed at testing the susceptibility of eleven economically important crops grown in the tropics to doses of these products applied before or at the onset of flowering.

\section{Materials and Methods}

Eleven experiments were conducted in pots kept outside under natural environmental conditions, from May 2009 to Jan. 2010, in Jaboticabal, state of São Paulo, Brazil. Local altitude is of $583 \mathrm{~m}$ above sea level, latitude is of $21^{\circ} 14^{\prime} 24^{\prime \prime}$ and longitude of $48^{\circ} 17^{\prime} 20^{\prime \prime}$. Each experiment was contained by one of the following cultivated plant species: peanuts (Arachis hypogaea L.), cotton (Gossypium hirsutum L.), potato (Solanum tuberosum L.), coffee (Coffea Arabica L.), beans (Phaseolus vulgaris L), sunflower (Helianthus annuиs L.), cassava (Manihot esculenta Crantz), rubber (Hevea brasiliensis Muell. Agr.), soybean (Glycine max L.), and grapes (Vitis vinifera L.). 
The experiment was a completely random design with five treatments (trinexapac-ethyl at 100 and $200 \mathrm{~g}$ $\mathrm{ha}^{-1}$, sulfometuron-methyl at 7.5 and $15 \mathrm{~g} \mathrm{ha}^{-1}$ and the control treatment) and four replications. The studied doses were established considering the products recommendations for sugar cane, corresponding to 50 and 100 $\%$ of the recommended dose for each one of them.

The experimental unit was an eight-liter pot filled with native soil $(63.7 \%$ of sand, $31.0 \%$ of clay, and 5.3 $\%$ of silt), sand, and an organic compound (organic material originating from plants), these components mixed in a $3: 1: 1$ proportion by volume. The soil was fertilized with $0.1 \mathrm{~g} \mathrm{~L}^{-1}$ of $\mathrm{N}$ (urea), $1.2 \mathrm{~g} \mathrm{~L}^{-1}$ of $\mathrm{P}$ (triple superphosphate), and $0.15 \mathrm{~g} \mathrm{~L}^{-1}$ of $\mathrm{K}$ (potassium chloride). Soybean plants were not fertilized.

Each treatment of cotton, peanuts, beans, sunflower, and soybean were sown with 10 seeds per pot. After seedling emergence, a thinning left three plants per pot, with the exception of sunflower, for which two plants were left. Coffee, citrus, rubber, and grape were grown from by transplanted seedlings. Potato plants were formed from tuber pieces and cassava plants from stem cuttings.

The plant regulators were applied at or soon before flowering by means of a backpack sprayer under constant (controlled by $\mathrm{CO}_{2}$ ) pressure applied at of $200 \mathrm{~L}$ $\mathrm{ha}^{-1}$. In Table 1, the date and hour of applications as well as the air relative humidity, the air temperature and the wind speed and also the number of plants per pot can be observed. The plant varieties, the sowing date and the evaluations made for each species are shown in Table 2.

The data were analyzed by the F-test of the analysis of variance and, when statistically significant values $(p<0.01$ or $p<0.05)$ were obtained, means were compared by the Tukey test $(p=0.05)$. SISVAR, a program for statistical analysis, was used.

\section{Results}

Seven days after application (DAA), trinexapacethyl at $200 \mathrm{~g} \mathrm{ha}^{-1}$ caused phytotoxic symptoms in peanut (average of $6 \%$ ) although these symptoms had dis- appeared on the 21 DAA (Table 3). At the lower dose, this product caused no visible injury to the plants. Sulfometuron-methyl caused more severe symptoms with mean values of $16 \%$ and $62 \%$ at 36 DAA for the doses of 7.5 and $15 \mathrm{~g} \mathrm{ha}^{-1}$, respectively. Nevertheless, the peanut plants, when submitted to $15 \mathrm{~g} \mathrm{ha}^{-1}$ of sulfometuron-methyl, accumulated the highest dry weight which differed only from the treatment with trinexapac-ethyl at $200 \mathrm{~g} \mathrm{ha}^{-1}$. Although the plants treated with sulfometuron at $15 \mathrm{~g} \mathrm{ha}^{-1}$ showed the highest amounts of dry weight, they produced less seed weight, different from the plants treated with trinexapac at $100 \mathrm{~g} \mathrm{ha}^{-1}(p<$ 0.05). When compared to the control treatment, plants treated with trinexapac at both doses showed no reduction in seed weight $(p<0.05)$. The yield components number of seeds per pot, number of pods per pot and number of seeds per pod were not significantly altered by the treatments.

Trinexapac-ethyl, at both studied doses, caused no toxicity symptoms in cotton plants (Table 4). However, sulfometuron-methyl, at both doses, caused visible injury to cotton plants, with mean values of $26 \%$ and $50 \%$ at $36 \mathrm{DAA}$ for the doses of 7.5 and $15 \mathrm{~g} \mathrm{ha}^{-1}$, respectively. When compared to the control, trinexapac had no effect on cotton plant growth, but sulfometuron did $(p<0.01)$. Cotton plants sprayed with sulfometuron were shorter and had lower dry weight than those of the control treatment $(p<0.01)$.

At 7 DAA, both plant regulators showed no toxicity symptoms on potato plants (Table 5). But, observations made on the 14 DAA, showed that sulfometuron at the doses of 7.5 and $15 \mathrm{~g}^{\text {ha }}{ }^{-1}$ caused symptoms classified as light $(5 \%)$ to moderate $(11 \%)$. However, on the $28 \mathrm{DAA}$, the symptoms had disappeared, as the plants recovered. Trinexapac, regardless of the dose, had no toxicity effects on the potato plants. As to the shoot dry weight and tubers fresh weight, the comparison between trinexapac at both doses and the control treatment had no differences $(p<0.01)$. The potato plants sprayed with $15 \mathrm{~g} \mathrm{ha}^{-1}$ of sulfometuron had lower tubers fresh weight although with higher shoot dry weight $(p<0.01)$.

Table 1 - Date and time, relative humidity, air temperature and wind velocity registered at the time of application of the growth regulators, number of plants per pot and development stages of the plants.

\begin{tabular}{lccccccc}
\hline Crop & Date & Time & $\begin{array}{c}\text { Relative } \\
\text { Humidity }\end{array}$ & Air temperature & $\begin{array}{c}\text { Wind } \\
\text { velocity }\end{array}$ & $\begin{array}{c}\text { Number of } \\
\text { plants per pot }\end{array}$ & Development stage \\
\hline Peanut & $07 / 30 / 09$ & $10 \mathrm{~h} 50-11 \mathrm{~h} 10$ & $74-70$ & $25.7-25.8$ & 1.0 & Three & $15 \mathrm{~cm}$ height, principal branch with 4 lateral branches \\
Cotton & $08 / 25 / 09$ & $16 \mathrm{~h} 00-16 \mathrm{~h} 10$ & $66-64$ & $23.5-24.4$ & 0.00 & Three & $30 \mathrm{~cm}$ height; 12 leaves (initial flowering) \\
Potato & $07 / 23 / 09$ & $09 \mathrm{~h} 10-10 \mathrm{~h} 10$ & $63-52$ & $22.2-26.4$ & $4.0-6.0$ & Four & $20 \mathrm{~cm}$ height, 8 leaves \\
Coffee & $11 / 24 / 09$ & $10 \mathrm{~h} 10-10 \mathrm{~h} 30$ & 74 & 29.3 & $1.0-1.5$ & One & $60-70 \mathrm{~cm}$ height \\
Citrus & $07 / 23 / 09$ & $09 \mathrm{~h} 10-10 \mathrm{~h} 10$ & $63-52$ & $22.2-26.4$ & $4.0-6.0$ & One & $70-90 \mathrm{~cm}$ height (flowering) \\
Bean & $11 / 24 / 09$ & $10 \mathrm{~h} 10-10 \mathrm{~h} 30$ & 74 & 29.3 & $1.0-1.5$ & Three & $35-45 \mathrm{~cm}$ height (initial flowering) \\
Sunflower & $07 / 30 / 09$ & $10 \mathrm{~h} 50-11 \mathrm{~h} 10$ & $74-70$ & $25.7-25.8$ & 1.0 & Two & $60 \mathrm{~cm}$ height, $8-10$ leaves \\
Cassava & $07 / 23 / 09$ & $09 \mathrm{~h} 10-10 \mathrm{~h} 10$ & $63-52$ & $22.2-26.4$ & $4.0-6.0$ & Three & $15 \mathrm{~cm}$ height, $8-10$ leaves \\
Rubber & $07 / 23 / 09$ & $09 \mathrm{~h} 10-10 \mathrm{~h} 10$ & $63-52$ & $22.2-26.4$ & $4.0-6.0$ & One & $65-85$ height, com new sprouting \\
Soybean & $10 / 20 / 09$ & $09 \mathrm{~h} 05-09 \mathrm{~h} 20$ & 71 & 23.9 & $2.0-4.0$ & Three & $32 \mathrm{~cm}$ height, $6-7$ trifoliate leaves (initial flowering) \\
Grape & $08 / 25 / 09$ & $16 h$ h0-16h10 & $66-64$ & $23.5-24.4$ & 0.00 & One & $130 \mathrm{~cm}$ height; 13 leaves
\end{tabular}


Table 2 - Variety, sowing and application date and description of the evaluated characteristics in each species.

\begin{tabular}{|c|c|c|c|c|c|}
\hline \multirow{2}{*}{ Crop } & \multirow{2}{*}{ Variety } & \multirow{2}{*}{ Sowing date } & \multicolumn{2}{|c|}{ Application } & \multirow{2}{*}{ Evaluations } \\
\hline & & & Date & $\mathrm{DAS}^{(1)}$ & \\
\hline Peanut & Runner IAC 886 & $07 / 02 / 2009$ & 07/30/2009 & 28 & $\begin{array}{c}\text { Injuries at 7, 14, } 21 \text { and } 36 \mathrm{DAA}^{(2)} \text {, shoot dry weight }{ }^{(3)} \text {, seed weight, number of seeds, } \\
\text { number of pods and number of seeds per pod at } 131 \mathrm{DAA}\end{array}$ \\
\hline Cotton & Delta Opal & $06 / 02 / 2009$ & 08/25/2009 & 84 & Injuries at 7, 16, 22, 29 and 36 DAA, height and shoot dry weight at 50 DAA \\
\hline Potato & Ágata & $06 / 16 / 2009$ & $07 / 23 / 2009$ & 37 & Injuries at 7, 14, 21 and 28 DAA, shoot dry weight e tubers fresh weight at 29 DAA \\
\hline Coffee & Rubi & $08 / 26 / 2009$ & $11 / 24 / 2009$ & 90 & $\begin{array}{c}\text { Injuries at 10, 15, } 22 \text { and } 36 \text { DAA, stem, shoot and total (stem + shoot) dry weight at } 44 \\
\text { DAA }\end{array}$ \\
\hline Citrus & Pêra Rio & $05 / 29 / 2009$ & 07/23/2009 & 55 & $\begin{array}{c}\text { Injuries at 7, 14, 21, } 28 \text { and } 43 \text { DAA, shoot dry weight at } 57 \text { DAA, height and number of } \\
\text { fruits at } 54 \text { DAA }\end{array}$ \\
\hline Bean & Pérola & $10 / 08 / 2009$ & $11 / 24 / 2009$ & 47 & $\begin{array}{c}\text { Injuries at 10, 15, } 22 \text { and } 36 \text { DAA, seed weight, number of seeds, number of pods and } \\
\text { number of seeds per pod at } 44 \text { DAA }\end{array}$ \\
\hline Sunflower & Catissol 01 & $06 / 30 / 2009$ & 07/30/2009 & 30 & $\begin{array}{c}\text { Injuries at 7, 14, } 21 \text { and } 36 \text { DAA, height at } 47 \text { DAA, number of heads per plant, shoot and } \\
\text { head dry weight at } 54 \text { DAA }\end{array}$ \\
\hline Cassava & Pão de açúcar & 05/06/2009 & 07/23/2009 & 78 & $\begin{array}{c}\text { Injuries at } 7,14,21,28 \text { and } 43 \text {, shoot and root } \text { ret }^{(4)} \text { dry weight at } 57 \text { DAA and number of } \\
\text { sprouts per pot at } 54 \text { DAA }\end{array}$ \\
\hline Rubber & RRIM 600 & 05/04/2009 & 07/23/2009 & 80 & Injuries at 7, 14, 21, 28 and 43 DAA, shoot dry weight at 57 DAA and height at 54 DAA \\
\hline Soybean & CD 214 RR & $09 / 18 / 2009$ & $10 / 20 / 2009$ & 32 & $\begin{array}{r}\text { Injuries at 7, 14, 21, } 30 \text { and } 37 \text { DAA, shoot and root dry weight, number and pod fresh } \\
\text { weight at } 49 \text { DAA }\end{array}$ \\
\hline Grape & Niagara Rosa & $07 / 22 / 2009$ & 08/25/2009 & 34 & $\begin{array}{r}\text { Injuries at 7, 16, 22, } 29 \text { and } 36 \mathrm{DAA} \text {, shoot dry weight, number and fresh weight of clusters } \\
\text { at } 50 \mathrm{DAA}\end{array}$ \\
\hline
\end{tabular}

${ }^{(1)}$ Day after sowing. (2) Day after application of the products. ${ }^{(3)}$ Plant shoot was collected and then dried in an oven at $50{ }^{\circ} \mathrm{C}$ until constant mass, then weighed. ${ }^{(4)}$ The roots were collected, washed and dried in an oven at $50^{\circ} \mathrm{C}$ until constant mass, then weighed.

Table 3 - Injuries in peanut plants at 7, 14, 21, and 36 days after application (DAA) of growth regulators, shoot dry weight, seed weight, number of seeds, number of pods and number of seeds per pods at 131 DAA.

\begin{tabular}{|c|c|c|c|c|c|c|c|c|c|c|}
\hline \multirow{2}{*}{$\begin{array}{l}\text { Growth } \\
\text { regulator }\end{array}$} & \multirow{2}{*}{ Dose } & \multicolumn{4}{|c|}{ Injuries (\%) - DAA } & \multirow{2}{*}{ Dry weight } & \multirow{2}{*}{$\begin{array}{l}\text { Seed } \\
\text { weight }\end{array}$} & \multirow{2}{*}{$\begin{array}{l}\text { Number of seeds per } \\
\text { pot }\end{array}$} & \multirow{2}{*}{$\begin{array}{l}\text { Number of pods per } \\
\text { pot }\end{array}$} & \multirow{2}{*}{$\begin{array}{l}\text { Number of seeds per } \\
\text { pod }\end{array}$} \\
\hline & & 7 & 14 & 21 & 36 & & & & & \\
\hline & $\mathrm{g} \mathrm{ha}^{-1}$ & & & & & \multicolumn{2}{|c|}{ - g per pot -} & & & \\
\hline Trinexapac & 100 & $0.0 a^{(2)}$ & $0.0 \mathrm{a}$ & $0.0 \mathrm{a}$ & $0.0 \mathrm{a}$ & $52.9 \mathrm{ab}$ & 80.3 a & $146.2 \mathrm{a}$ & $102.5 \mathrm{a}$ & $1.4 \mathrm{a}$ \\
\hline Trinexapac & 200 & $6.2 \mathrm{a}$ & $5.0 \mathrm{a}$ & $0.0 \mathrm{a}$ & $0.0 \mathrm{a}$ & $39.1 \mathrm{~b}$ & $69.2 a b$ & $122.0 \mathrm{a}$ & $82.8 \mathrm{a}$ & $1.5 \mathrm{a}$ \\
\hline Sulfometuron & 7.5 & $17.5 b$ & $27.5 b$ & $30.0 \mathrm{~b}$ & $16.2 \mathrm{a}$ & $57.0 \mathrm{ab}$ & $70.6 a b$ & $129.2 \mathrm{a}$ & $95.2 \mathrm{a}$ & $1.4 \mathrm{a}$ \\
\hline Sulfometuron & 15 & $18.8 \mathrm{~b}$ & $51.2 \mathrm{c}$ & $60.0 \mathrm{c}$ & $62.5 b$ & $60.1 \mathrm{a}$ & $56.3 b$ & $115.5 \mathrm{a}$ & $87.8 \mathrm{a}$ & $1.3 \mathrm{a}$ \\
\hline Control & - & $0.0 \mathrm{a}$ & $0.0 \mathrm{a}$ & $0.0 \mathrm{a}$ & $0.0 \mathrm{a}$ & $51.7 \mathrm{ab}$ & $68.6 a b$ & $136.2 \mathrm{a}$ & $96.5 \mathrm{a}$ & $1.4 \mathrm{a}$ \\
\hline $\mathrm{F}$ & & $31.0^{* *}$ & $57.5^{* *}$ & $48.0^{* *}$ & $52.1^{* *}$ & $3.8^{*}$ & 4.7 & 1.5 & 2.0 & 0.7 \\
\hline CV (\%) & & 38.7 & 34.4 & 43.0 & 47.6 & 15.8 & 11.5 & 15.0 & 11.8 & 11.2 \\
\hline LSD & & 7.2 & 12.8 & 16.9 & 16.4 & 18.0 & 17.3 & 42.6 & 23.7 & 0.3 \\
\hline
\end{tabular}

** * Significant at $1 \%$ and $5 \%$, respectively, by F test of variance analyses. ${ }^{(2)}$ Means followed by the same letter are not different (Tukey test, $\left.p<0.05\right)$.

Trinexapac caused no visible injury to bean plants whereas sulfometuron caused damages classified as severe (mean values of $39 \%$ and $59 \%$ on the $10^{\text {th }}$ DAA for doses of 7.5 and $15 \mathrm{~g} \mathrm{ha}^{-1}$, respectively) although these symptoms faded away with time, indicating that the plants could recover from the damages (Table 6). When seed weight $(p<0.05)$ and number of seeds per plant $(p<0.01)$ were considered, in comparison with the control treatment, only trinexapac at $100 \mathrm{~g} \mathrm{ha}^{-1}$ caused no reduction in plant performance. For number of pods, the results of trinexapac at both doses are not different from those of the control $\mid p<$
0.01). Number of seeds per pod, when comparisons with the control treatment are made, was not affected by any of the treatments.

Trinexapac, in comparison with the control treatment, independently of the dose, caused no negative effect on any of the evaluated characteristics sunflower plants. Nevertheless, plants were visibly damaged by both doses of sulfometuron, with injuries, classified as severe, caused dramatic reductions in plant growth (Table 7). These reductions are observed in plant height, in the shoot dry weight, and in flower head dry weight $(p$ $<0.01)$. 
Table 4 - Injuries in cotton plants at 7, 16, 22, 29, and 36 days after application (DAA) of the growth regulators, height and shoot dry weight of cotton plants at 50 DAA.

\begin{tabular}{|c|c|c|c|c|c|c|c|c|}
\hline \multirow{2}{*}{$\begin{array}{l}\text { Growth } \\
\text { regulator }\end{array}$} & \multirow{2}{*}{ Dose } & \multicolumn{5}{|c|}{ Injuries (\%) - DAA } & \multirow{2}{*}{ Plant height } & \multirow{2}{*}{ Dry weight } \\
\hline & & 7 & 16 & 22 & 29 & 36 & & \\
\hline & $\mathrm{g} \mathrm{ha}^{-1}$ & & & & & & $\mathrm{~cm}$ & g per plant \\
\hline Trinexapac & 100 & $0.0 a^{(1)}$ & $0.0 \mathrm{a}$ & $0.0 \mathrm{a}$ & $0.0 \mathrm{a}$ & $0.0 \mathrm{a}$ & $55.2 \mathrm{a}$ & 19.2 a \\
\hline Trinexapac & 200 & $0.0 \mathrm{a}$ & $0.0 \mathrm{a}$ & $0.0 \mathrm{a}$ & $0.0 \mathrm{a}$ & $0.0 \mathrm{a}$ & $54.4 \mathrm{a}$ & 19.4 a \\
\hline Sulfometuron & 7.5 & $16.2 \mathrm{~b}$ & $22.5 \mathrm{~b}$ & $27.5 b$ & $27.5 b$ & $26.2 \mathrm{~b}$ & $35.6 \mathrm{~b}$ & $12.4 \mathrm{~b}$ \\
\hline Sulfometuron & 15 & $18.8 b$ & $46.2 \mathrm{c}$ & $50.0 \mathrm{c}$ & $57.5 c$ & $50.0 \mathrm{c}$ & $33.6 \mathrm{~b}$ & $10.2 \mathrm{~b}$ \\
\hline Control & - & $0.0 \mathrm{a}$ & $0.0 \mathrm{a}$ & $0.0 \mathrm{a}$ & $0.0 \mathrm{a}$ & $0.0 \mathrm{a}$ & $52.8 \mathrm{a}$ & $18.5 \mathrm{a}$ \\
\hline $\mathrm{F}$ & & $148.2^{* *}$ & $86.8^{* *}$ & $94.8^{* *}$ & $52.4^{* *}$ & $33.1^{\text {** }}$ & $89.7^{* *}$ & $20.4^{* *}$ \\
\hline CV (\%) & & 22.6 & 32.2 & 30.0 & 41.6 & 51.3 & 4.9 & 12.0 \\
\hline LSD & & 3.4 & 9.7 & 10.2 & 15.4 & 17.1 & 5.0 & 4.2 \\
\hline
\end{tabular}

${ }^{* *}$ Significant at $1 \%$ probability by $\mathrm{F}$ test of variance analyses. ${ }^{(1)}$ Means followed by the same letter are not different (Tukey test, $p<0.05$ ).

Table 5 - Injuries in potato plants at 7, 14, 21 and 28 days after application (DAA) of growth regulators, shoot dry weight per pot and tuber fresh weight per pot at 29 DAA.

\begin{tabular}{|c|c|c|c|c|c|c|c|}
\hline \multirow{2}{*}{$\begin{array}{l}\text { Growth } \\
\text { regulator }\end{array}$} & \multirow{2}{*}{ Dose } & \multicolumn{4}{|c|}{ Injuries (\%) - DAA } & \multirow{2}{*}{ Dry weight } & \multirow{2}{*}{ Tuber weight } \\
\hline & & 7 & 14 & 21 & 28 & & \\
\hline & $\mathrm{g} \mathrm{ha}^{-1}$ & & & & & 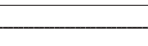 & 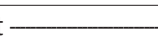 \\
\hline Trinexapac & 100 & 0.0 & $0.0 a^{(1)}$ & $0.0 \mathrm{a}$ & 0.0 & $10.8 b$ & $376.9 \mathrm{ab}$ \\
\hline Trinexapac & 200 & 0.0 & $0.0 \mathrm{a}$ & $0.0 \mathrm{a}$ & 0.0 & $11.5 \mathrm{~b}$ & $355.2 \mathrm{abc}$ \\
\hline Sulfometuron & 7.5 & 0.0 & $5.0 \mathrm{~b}$ & $2.5 \mathrm{a}$ & 0.0 & $12.7 \mathrm{ab}$ & 308.3 bc \\
\hline Sulfometuron & 15 & 0.0 & $11.2 \mathrm{c}$ & $1.2 \mathrm{a}$ & 0.0 & $15.8 \mathrm{a}$ & $251.9 \mathrm{c}$ \\
\hline Control & - & 0.0 & $0.0 \mathrm{a}$ & $0.0 \mathrm{a}$ & 0.0 & $10.8 b$ & $452.6 \mathrm{a}$ \\
\hline $\mathrm{F}$ & & - & $21.5^{\text {** }}$ & 1.7 & - & $7.1^{* *}$ & $9.6^{* *}$ \\
\hline CV (\%) & & - & 65.9 & 27.7 & - & 12.8 & 13.9 \\
\hline LSD & & - & 4.7 & 3.7 & - & 3.4 & 105.9 \\
\hline
\end{tabular}

${ }^{* *}$ Significant at $1 \%$ probability by $\mathrm{F}$ test of variance analyses. ${ }^{(1)}$ Means followed by the same letter are not different (Tukey test, $p<0.05$ ).

Table 6 - Injuries in bean plants at 10, 15, 22 and 36 days after application (DAA) of growth regulators, seed weight, number of seeds, number of pods, number of seeds per pod at 44 DAA.

\begin{tabular}{|c|c|c|c|c|c|c|c|c|c|}
\hline \multirow{2}{*}{$\begin{array}{l}\text { Growth } \\
\text { regulator }\end{array}$} & \multirow{2}{*}{ Dose } & \multicolumn{4}{|c|}{ Injuries (\%) - DAA } & \multirow[t]{2}{*}{ Seed weight } & \multirow{2}{*}{$\begin{array}{c}\text { Number of seeds per } \\
\text { plant }\end{array}$} & \multirow{2}{*}{$\begin{array}{l}\text { Number of pods per } \\
\text { plant }\end{array}$} & \multirow{2}{*}{$\begin{array}{l}\text { Number of seeds per } \\
\text { pod }\end{array}$} \\
\hline & & 10 & 15 & 22 & 36 & & & & \\
\hline & $\mathrm{g} \mathrm{ha}^{-1}$ & & & & & g per pot & & & \\
\hline Trinexapac & 100 & $0.0 a^{(1)}$ & $0.0 \mathrm{a}$ & $0.0 \mathrm{a}$ & $0.0 \mathrm{a}$ & $3.2 \mathrm{ab}$ & $15.3 \mathrm{ab}$ & $4.7 \mathrm{a}$ & $3.3 \mathrm{a}$ \\
\hline Trinexapac & 200 & $0.0 \mathrm{a}$ & $0.0 \mathrm{a}$ & $0.0 \mathrm{a}$ & $0.0 \mathrm{a}$ & $2.6 \mathrm{~b}$ & $14.1 \mathrm{~b}$ & $4.5 \mathrm{a}$ & $3.2 \mathrm{a}$ \\
\hline Sulfometuron & 7.5 & $38.8 b$ & $31.2 \mathrm{~b}$ & $23.8 \mathrm{~b}$ & $0.0 \mathrm{a}$ & $0.1 \mathrm{c}$ & $1.2 \mathrm{c}$ & $0.7 \mathrm{~b}$ & $2.5 \mathrm{a}$ \\
\hline Sulfometuron & 15 & $58.8 \mathrm{c}$ & $58.8 \mathrm{c}$ & $66.2 \mathrm{c}$ & $30.0 \mathrm{~b}$ & $0.0 \mathrm{c}$ & $0.0 \mathrm{c}$ & $0.3 \mathrm{~b}$ & $0.0 \mathrm{a}$ \\
\hline Control & - & $0.0 \mathrm{a}$ & $0.0 \mathrm{a}$ & $0.0 \mathrm{a}$ & $0.0 \mathrm{a}$ & $4.6 \mathrm{a}$ & $20.2 \mathrm{a}$ & $5.9 \mathrm{a}$ & $3.5 \mathrm{a}$ \\
\hline$F$ & & $523.2^{\text {** }}$ & $78.4^{* *}$ & 93.0 ** & $24.0^{* *}$ & $37.1^{*}$ & $58.4^{* *}$ & $37.2^{* *}$ & 2.1 \\
\hline CV (\%) & & 12.4 & 33.3 & 33.3 & 91.3 & 31.2 & 23.3 & 25.9 & 79.8 \\
\hline LSD & & 5.3 & 13.1 & 13.1 & 12.0 & 1.4 & 5.2 & 1.8 & 4.3 \\
\hline
\end{tabular}

${ }^{* *}$ Significant at $1 \%$ probability by $\mathrm{F}$ test of variance analyses. ${ }^{(1)}$ Means followed by the same letter are not different (Tukey test, $p<0.05$ ).

Trinexapac, in comparison with the control treatment, at both doses, caused no visible damage to soybean plants (Table 8). Sulfometuron, caused severe injury to the plants with mean values of $74 \%$ and $96 \%$ at 37 DAA for the doses of 7.5 and $15 \mathrm{~g} \mathrm{ha}^{-1}$. Sulfometuron at the dose of $7.5 \mathrm{~g} \mathrm{ha}^{-1}$, though, had no effect on root dry weight $(p<0.01)$.

Grape plants showed no damage signs when they were treated with trinexapac at the dose of $100 \mathrm{~g} \mathrm{ha}^{-1}$ (Table 9); at $200 \mathrm{~g} \mathrm{ha}^{-1}$, though, trinexapac caused inju- ries classified from light $(1 \%)$ to moderate $(9 \%)$. Sulfometuron, in observations made 29 DAA, caused damages classified as severe with mean values between $49 \%$ to $51 \%$ for the doses of 7.5 and $15 \mathrm{~g} \mathrm{ha}^{-1}$, respectively. The plants sprayed with trinexapac had values for shoot dry weight, number and fresh weight of clusters statistically similar to those found for the control treatment.

Trinexapac and sulfometuron, at both doses, had no effect on any of the evaluated plant characteristics of coffee, citrus, cassava, and rubber. 
Table 7 - Injuries in sunflower plants at 7, 14, 21 and 36 days after application (DAA) of growth regulators, plant height at 47 DAA, shoot and head weight, number of heads per plant, at 54 DAA.

\begin{tabular}{|c|c|c|c|c|c|c|c|c|c|}
\hline \multirow{2}{*}{$\begin{array}{l}\text { Growth } \\
\text { regulator }\end{array}$} & \multirow{2}{*}{ Dose } & \multicolumn{4}{|c|}{ Injuries (\%) - DAA } & \multirow{2}{*}{ Plant height } & \multicolumn{2}{|c|}{ Dry weight } & \multirow{2}{*}{ Number of heads per plant } \\
\hline & & 7 & 14 & 21 & 36 & & Shoot & Head & \\
\hline & $\mathrm{g} \mathrm{ha}^{-1}$ & & & & & $\mathrm{~cm}$ & \multicolumn{2}{|c|}{ g per plant } & \\
\hline Trinexapac & 100 & $0.0 a^{(1)}$ & $0.0 \mathrm{a}$ & $0.0 \mathrm{a}$ & $0.0 \mathrm{a}$ & $120.8 \mathrm{a}$ & $33.1 \mathrm{a}$ & $11.1 \mathrm{a}$ & $0.8 a b$ \\
\hline Trinexapac & 200 & $0.0 \mathrm{a}$ & $0.0 \mathrm{a}$ & $0.0 \mathrm{a}$ & $0.0 \mathrm{a}$ & $112.1 \mathrm{a}$ & $25.8 \mathrm{a}$ & $15.1 \mathrm{a}$ & $1.4 \mathrm{a}$ \\
\hline Sulfometuron & 7.5 & $51.2 \mathrm{~b}$ & $70.0 \mathrm{~b}$ & $83.8 \mathrm{~b}$ & $63.8 \mathrm{~b}$ & $62.9 b$ & $13.4 \mathrm{~b}$ & $3.3 b$ & $0.5 a b$ \\
\hline Sulfometuron & 15 & $57.5 b$ & $80.0 \mathrm{c}$ & $92.5 b$ & $97.5 \mathrm{c}$ & $38.1 \mathrm{c}$ & $9.3 \mathrm{~b}$ & $0.0 \mathrm{~b}$ & $0.0 \mathrm{~b}$ \\
\hline Control & - & $0.0 \mathrm{a}$ & $0.0 \mathrm{a}$ & $0.0 \mathrm{a}$ & $0.0 \mathrm{a}$ & $125.5 \mathrm{a}$ & $27.8 \mathrm{a}$ & $12.6 \mathrm{a}$ & $0.9 a b$ \\
\hline $\mathrm{F}$ & & $155.7^{* *}$ & $680.0^{* *}$ & $477.8^{* *}$ & $66.3^{* *}$ & $67.6^{* *}$ & $12.7^{* *}$ & $16.3^{* *}$ & $5.8^{* *}$ \\
\hline CV (\%) & & 22.0 & 10.5 & 12.6 & 34.8 & 10.4 & 25.9 & 38.0 & 59.8 \\
\hline LSD & & 10.5 & 6.9 & 9.7 & 24.5 & 20.8 & 12.4 & 7.0 & 0.9 \\
\hline
\end{tabular}

${ }^{*}$ * Significant at $1 \%$ probability by $\mathrm{F}$ test of variance analyses. ${ }^{(1)}$ Means followed by the same letter are not different (Tukey test, $p<0.05$ ).

Table 8 - Injuries in soybean plants at 7, 14, 21, 30 and 37 days after application (DAA) of growth regulators, shoot and root dry weight, number of pods and pod fresh weight at 49 DAA.

\begin{tabular}{|c|c|c|c|c|c|c|c|c|c|c|}
\hline \multirow{2}{*}{$\begin{array}{l}\text { Growth } \\
\text { regulator }\end{array}$} & \multirow{2}{*}{ Dose } & \multicolumn{5}{|c|}{ Injuries (\%) - DAA } & \multicolumn{2}{|c|}{ Dry weight } & \multirow{2}{*}{$\begin{array}{l}\text { Number of pods } \\
\text { per plant }\end{array}$} & \multirow{2}{*}{ Pod fresh weight } \\
\hline & & 7 & 14 & 21 & 30 & 37 & Shoot & Root & & \\
\hline & $\mathrm{g} \mathrm{ha}^{-1}$ & & & & & & \multicolumn{2}{|c|}{ - g per plant - } & & g per plant \\
\hline Trinexapac & 100 & $0.0 a^{(1)}$ & $0.0 \mathrm{a}$ & $0.0 \mathrm{a}$ & $0.0 \mathrm{a}$ & $0.0 \mathrm{a}$ & $11.6 \mathrm{ab}$ & $32.1 \mathrm{a}$ & $16.8 \mathrm{a}$ & $2.4 \mathrm{a}$ \\
\hline Trinexapac & 200 & $0.0 \mathrm{a}$ & $0.0 \mathrm{a}$ & $0.0 \mathrm{a}$ & $0.0 \mathrm{a}$ & $0.0 \mathrm{a}$ & $12.9 \mathrm{a}$ & $41.6 \mathrm{a}$ & $17.8 \mathrm{a}$ & $2.2 \mathrm{a}$ \\
\hline Sulfometuron & 7.5 & $27.5 b$ & $40.0 \mathrm{~b}$ & $48.8 \mathrm{~b}$ & $71.2 \mathrm{~b}$ & $73.8 \mathrm{~b}$ & $8.5 b$ & $23.2 a b$ & $2.8 \mathrm{~b}$ & $0.2 \mathrm{~b}$ \\
\hline Sulfometuron & 15 & $23.8 b$ & $51.2 \mathrm{~b}$ & $66.2 c$ & $95.0 \mathrm{c}$ & $96.2 \mathrm{c}$ & $3.9 c$ & $7.4 \mathrm{~b}$ & $0.0 \mathrm{~b}$ & $0.0 \mathrm{~b}$ \\
\hline Control & - & $0.0 \mathrm{a}$ & $0.0 \mathrm{a}$ & $0.0 \mathrm{a}$ & $0.0 \mathrm{a}$ & $0.0 \mathrm{a}$ & $13.1 \mathrm{a}$ & 40.3 a & 20.7 a & $3.0 \mathrm{a}$ \\
\hline $\mathrm{F}$ & & $48.9^{* *}$ & $57.4^{* *}$ & $105.2^{* *}$ & $1,083.0^{* *}$ & $1,784.6^{* *}$ & $15.3^{\text {** }}$ & $6.4^{* *}$ & $96.9^{* *}$ & $15.0^{* *}$ \\
\hline CV (\%) & & 39.3 & 36.6 & 27.2 & 8.5 & 6.6 & 19.9 & 38.7 & 16.6 & 45.5 \\
\hline LSD & & 8.8 & 14.6 & 13.8 & 6.1 & 4.9 & 4.4 & 24.4 & 4.2 & 1.5 \\
\hline
\end{tabular}

${ }^{* *}$ Significant at $1 \%$ probability by $F$ test of variance analyses. ${ }^{(1)}$ Means followed by the same letter are not different (Tukey test, $p<0.05$ ).

Table 9 - Injuries in grape plants at 7, 16, 22, 29 and 36 days after application (DAA) of growth regulators, shoot dry weight, number and fresh weight of grape clusters at 50 DAA.

\begin{tabular}{|c|c|c|c|c|c|c|c|c|c|}
\hline \multirow{2}{*}{$\begin{array}{l}\text { Growth } \\
\text { regulator }\end{array}$} & \multirow{2}{*}{ Dose } & \multicolumn{5}{|c|}{ Injuries (\%) - DAA } & \multirow{2}{*}{ Dry weight } & \multirow{2}{*}{$\begin{array}{l}\text { Number of clusters } \\
\text { per plant }\end{array}$} & \multirow{2}{*}{$\begin{array}{c}\text { Cluster fresh } \\
\text { weight }\end{array}$} \\
\hline & & 7 & 16 & 22 & 29 & 36 & & & \\
\hline & $\mathrm{g} \mathrm{ha}^{-1}$ & & & & & & g per plant & & g per plant \\
\hline Trinexapac & 100 & $0.0 a^{(1)}$ & $0.0 \mathrm{a}$ & $0.0 \mathrm{a}$ & $0.0 \mathrm{a}$ & $0.0 \mathrm{a}$ & $15.4 \mathrm{a}$ & $2.0 \mathrm{ab}$ & $43.1 \mathrm{ab}$ \\
\hline Trinexapac & 200 & $1.2 \mathrm{a}$ & $5.0 \mathrm{a}$ & $8.8 b$ & $6.2 \mathrm{~b}$ & $2.5 \mathrm{a}$ & $15.4 \mathrm{a}$ & $2.5 \mathrm{ab}$ & $54.5 \mathrm{a}$ \\
\hline Sulfometuron & 7.5 & $5.0 \mathrm{~b}$ & $12.5 \mathrm{~b}$ & $26.2 \mathrm{c}$ & $49.4 \mathrm{c}$ & $46.9 \mathrm{~b}$ & $10.8 \mathrm{~b}$ & $0.5 \mathrm{~b}$ & $0.5 b$ \\
\hline Sulfometuron & 15 & $5.0 \mathrm{~b}$ & $12.5 \mathrm{~b}$ & $22.5 \mathrm{c}$ & $51.2 \mathrm{c}$ & $51.2 \mathrm{~b}$ & $9.8 \mathrm{~b}$ & $0.5 b$ & $0.9 \mathrm{~b}$ \\
\hline Control & - & $0.0 \mathrm{a}$ & $0.0 \mathrm{a}$ & $0.0 \mathrm{a}$ & $0.0 \mathrm{a}$ & $0.0 \mathrm{a}$ & $11.1 \mathrm{ab}$ & $2.8 \mathrm{a}$ & $47.7 \mathrm{a}$ \\
\hline $\mathrm{F}$ & & 21.0 ** & $23.6^{* *}$ & $56.4^{* *}$ & $1,002.3^{* *}$ & $261.4^{* *}$ & $3.9^{*}$ & $3.6^{*}$ & $8.4^{* *}$ \\
\hline CV (\%) & & 49.7 & 43.0 & 28.6 & 7.8 & 16.3 & 21.8 & 69.5 & 62.3 \\
\hline DMS & & 2.4 & 5.6 & 7.2 & 3.2 & 6.3 & 5.2 & 2.2 & 40.0 \\
\hline
\end{tabular}

${ }^{\star \star, *}$ Significant at $1 \%$ and $5 \%$ probability, respectively, by $\mathrm{F}$ test of variance analyses. ${ }^{(1)}$ Means followed by the same letter are not different (Tukey test, $\left.p<0.05\right)$.

\section{Discussion}

The results herein reported support the hypothesis that the phytotoxic effects of trinexapac and sulfometuron are species dependent (some species are more susceptible than others) and that sulfumeturon is more toxic than trinexapac. This variability seems to be explained by the mode of action of the product in the plant and also by the capacity of the plant to detoxify themselves or metabolize the product.

Sulfometuron-methyl belongs to the sulfonylurea chemical group, and its primary site of action consists in the inhibiting of the enzyme acetolactate synthase (ALS) or the enzyme acetohydroxyacid synthase (AHAS) thus interfering in synthesis of the essential amino acids valine, leucine, and isoleucine (Cox, 2002; Zhou et al., 2007). In the USA, this active ingredient is used mostly 
in nonagricultural situations and as a selective herbicide on conifer plantations, hardwood plantations, and turf (Cox, 2002) at high doses [up to $52.5 \mathrm{~g} \mathrm{ha}^{-1}$ (Ahrens, 1994)]. In Brazil, the doses used in sugar cane crops as a plant ripener are not higher than $15 \mathrm{~g} \mathrm{ha}^{-1}$ (MAPA, 2011a).

The phytotoxic effect of sulfometuron for some crops has been reported. Novo and Miranda Filho (2006) reported decrements in the total number of tubers and in the fresh weight of normal tubers when increasing (from 0 to $15 \mathrm{~g} \mathrm{ha}^{-1}$ ) doses of sulfometuron were applied to the plants. In another study, soil residues of sulfometuron larger than 240 parts per trillion caused distorted tubers with cracks and folds (Hutchinson et al., 2007). Olszyk et al. (2010) also observed that treatments with low levels of sulfometuron caused large reductions in tuber fresh weight. The effect on tuber fresh weight was due to reduced tuber size, insofar as it did not affect the number of tubers. In addition to that, sulfometuron had much less effect on potato plant vegetative growth than on tuber production. The importance of measuring tuber production and not just vegetative shoot growth as an indicator of risk from low levels of herbicides was highlighted by the response of the young potato plants to sulfometuron. There was a much greater effect on tuber yield than on vegetative growth, measured especially as shoot dry weight (Olszyk et al., 2010). In this study, the results indicated that the sulfometuron had an influence on the source-sink relation of the potato plant and this caused the partition process to be unbalanced resulting in an accumulation of photosynthates in the plant aerial part.

Soybean plants exposed to $5.25 \mathrm{~g} \mathrm{ha}^{-1}$ of sulfometuron showed dramatic reductions in seed weight, seed number, pod number, and pod weight (Pfleeger et al., 2011). However, peas were generally more sensitive to low levels of sulfometuron than potatoes or soybeans, and pea reproductive endpoints were usually more sensitive than vegetative ones (Pfleeger et al., 2011). In the life cycle of a pea, the earlier its exposition occurred, the lower the concentration able to impact the fresh pea weight. Besides this, there are reports of phytotoxic effects of sulfometuron in plants of cherry, canola, sunflower, and smartweed (Cox, 2002). Cox (2002) reported that an investigation by the Idaho Department of Agriculture recently concluded that several million dollars worth of crops were damaged by wind transport from an aerial sulfometuron application made by the Bureau of Land Management to kill cheatgrass following a wildfire. Over a hundred farmers and ranchers reported damage on over 100,000 acres.

Data in the literature confirm those reported in this paper because the sulfometuron caused severe visual injuries in seven of the 11 species studied, them affecting plant growth. Dose effect was also observed for peanut plants, the plants treated with $7.5 \mathrm{~g} \mathrm{ha}^{-1}$ were less affected than those receiving higher doses. Only peanut showed this response to dose. Bean, cotton, potato, soybean, sun- flower, and grape plants showed extreme sensitivity to sulfometuron, even when submitted to the lowest dose. Coffee, citrus, cassava, and rubber plants, under the evaluated conditions, even when exposed to the highest dose of sulfometuron, showed no visible injury. Changes related to absorption, translocation, site of action, metabolism or even compartmentalization of sulfometuron by plants may explain the variability of response of species to action of the product.

Trinexapac is an acylcyclehexanedione (Caldas et al., 2009; Rademacher, 2000) that, in Poaceae species, such as wheat and rye, causes a reduction in the internode length and, consequently, in plant height. This has been used to reduce plant lodging (MAPA, 2011b; Espindula et al., 2009; Zagonel and Fernandes, 2007). This effect is explained by the trinexapac action on the synthesis of gibberellins, starting from the $\mathrm{GA}_{12}$ aldehyde which, among other functions, is responsible for cell elongation (Caldas et al., 2009; Dalley and Richard Junior, 2010; Rademacher, 2000). As a consequence of becoming incapable of synthesizing active gibberellins, the plants start to synthesize and accumulate biologically less efficient gibberellins and this causes reductions in cell elongation (Rademacher, 2000; Taiz and Zeiger, 2006). In dicotyledons, this inhibition is not so strong, thus justifying the selectivity of trinexapac to plants of that group (Rademacher, 2000).

In addition to the anti-lodging effect in cereals and increments on the sucrose production in sugar cane, the inhibitors of gibberellins synthesis retard the growth and flowering of grasses (Costa et al., 2009; Freitas et al., 2002) and the fructification of decidual fruit plants. Mouco et al. (2010) verified that spraying trinexapac at high doses ( 1.0 or a sequential value of 0.5 plus $0.5 \mathrm{~g}$ per plant) on 'Tommy Atkins' mango plants was efficient in controlling the vegetative growth of the branches. This effect would not be observed in the field simply by the occurrence of drift or technical procedures incorrectly applied, due to the low concentration per plant verified in this situation. Taking a population of 300,000 soybean plants per hectare the equivalent concentration per plant would be of 0.33 and $0.67 \mathrm{mg}$ per plant in a direct spraying of trinexapac at doses of 100 or $200 \mathrm{~g} \mathrm{ha}^{-1}$. These values are well below those used in orchards for retarding vegetative growth and consequent management of floral induction.

Trinexapac was selective to the studied species with the exception of beans which was sensitive to the higher dose of the product. In that concentration, seed weight underwent reduction compared to control treatment. The less aggressive effect of trinexapac as compared to that of sulfometuron was expected, because the trinexapac is indeed classified as a plant regulator and not a herbicide. This product cause plant height reductions in species of the Poaceae family but without effect on grain yield (Zagonel and Fernandes, 2007). Trinexapac, at 100 and $200 \mathrm{~g} \mathrm{ha}^{-1}$, was considered safe for aerial spraying in sugarcane crops causing no risks to the tested dicotyledonous species even if drifting or 
technical mistakes during the product spraying occur. As already mentioned, bean plants, if exposed to the higher dose, will exhibit injury signs.

We could not find any reports in the literature about phytotoxic effects of trinexapac on the vegetative or reproductive development on dicotyledonous crops. In addition, little information is available concerning the effects of sulfometuron on non target crops. It is a potent herbicide whose toxic action in sensitive plants is being currently under study in others regions with temperature climates such as North America and Europe. However, in Brazil and other tropical countries, this subject has scarcely been considered by researchers. Other similar research is needed, ideally under field conditions. The objectives of such investigations should be exposure at various concentrations and the application at different developmental stages.

\section{Conclusions}

Trinexapac-ethyl, at the doses of 100 and $200 \mathrm{~g}$ $\mathrm{ha}^{-1}$, showed phytotoxic selectivity to peanuts, cotton, potato, coffee, citrus, sunflower, cassava, rubber, soybean, and grape. At the lowest dose (100 $\left.\mathrm{g} \mathrm{ha}^{-1}\right)$, it was selective for bean. Sulfometuron-methyl, at the dose of $7.5 \mathrm{~g} \mathrm{ha}^{-1}$, was selective for peanuts and, at the two studied doses (7.5 and $15 \mathrm{~g} \mathrm{ha}^{-1}$ ), it was selective for coffee, citrus, cassava, and rubber.

\section{References}

Ahrens, W.H. 1994. Herbicide Handbook. Weed Science Society of America, Champaign, IL, USA.

Caldas, L.S.; Machado, L.L.; Caldas, S.C.; Campos, M.L.; Caldas, J.A.; Pharis, R.P.; Pereira-Netto, A.B. 2009. Growth-active gibberellins overcome the very slow shoot growth of Hancornia speciosa, an important fruit tree from the Brazilian "Cerrado". Trees 23: 1229-1235.

Castro, P.R.C. 1999. Chemicals ripeners in sugarcane. Saccharum 1: 12-16 (in Portuguese).

Costa, N.V.; Martins, D.; Rodella, R.A.; Rodrigues, A.C.P.; Cardoso, L.A. 2009. Morphological characteristics of turf grasses in response to trinexapac-ethyl application. Planta Daninha 27: 113-122 (in Portuguese, with abstract in English).

Cox, C. 2002. Sulfometuron methyl (Oust). Journal of Pesticide Reform 22: 15-20.

Dalley, C.D.; Richard Junior, E.P. 2010. Herbicides as ripeners for sugarcane. Weed Science 58: 329-333.
Espindula, M.C.; Rocha, V.S.; Grossi, J.A.S.; Souza, M.A.; Souza, L.T.; Favarato, L.F. 2009. Use of growth retardants in wheat. Planta Daninha 27: 379-387.

Freitas, F.C.L.; Ferreira, L.R.; Silva, A.A.; Barbosa, J.G.; Miranda, G.V. 2002. Effects of trinexapac-ethyl on the growth and flowering of the bahiagrass. Planta Daninha 20: 477-486 (in Portuguese, with abstract in English).

Hutchinson, P.J.; Morishita, D.W.; Price, W.J. 2007. Season-long dose-response of potato to sulfometuron. Weed Science 55: 521-527.

Ministério da Agricultura, Pecuária e Abastecimento [MAPA]. AGROFIT - Sistema de Agrotóxicos Fitossanitários. 2011a. Available at: http://extranet.agricultura.gov.br/agrofit_cons/ principal_agrofit_cons. [Accessed May 7, 2011] (in Portuguese).

Ministério da Agricultura, Pecuária e Abastecimento [MAPA]. AGROFIT - Sistema de Agrotóxicos Fitossanitários. 2011b. Available at: http://extranet.agricultura.gov.br/agrofit_cons/ principal_agrofit_cons. [Accessed May 7, 2011] (in Portuguese).

Mouco, M.A.C.; Ono, E.O.; Rodrigues, J.D. 2010. Synthesis inhibitors of gibberellins and mango 'Tommy Atkins' seedlings growth. Ciência Rural 40: 273-279 (in Portuguese, with abstract in English).

Novo, M.C.S.S.; Miranda Filho, H.S. 2006. Effect of sulfonylurea herbicides on tuberization of two potato cultivars. Planta Daninha 24: 115-121 (in Portuguese, with abstract in English).

Olszyk, D.; Pfleeger, T.; Henry Lee, E.; Plocher, M. 2010. Potato (Solanum Tuberosum) greenhouse tuber production as an assay for asexual reproduction effects from herbicides. Environmental Toxicology and Chemistry 29: 111-121.

Pfleeger, T.; Olszyk, D.; Henry Lee, E.; Plocher, M. 2011. Comparing effects of low levels of herbicides on greenhouse and field-grown potatoes (Solanum tuberosum L.), soybeans (Glycine max L.), and peas (Pisum sativum L.). Environmental Toxicology and Chemistry 30: 455-468.

Rademacher, W. 2000. Growth retardants: effects on gibberellin biosynthesis and other metabolic pathways. Annual Review of Plant Physiology and Plant Molecular Biology 51: 501-531.

Taiz, L.; Zeiger, E. 2006. Plant Physiology. Sinauer, Sunderland, MA, USA.

Zagonel, J.; Fernandes, E.C. 2007. Rates and application times of growth reducer affecting wheat cultivars at two nitrogen rates. Planta Daninha 25: 331-339 (in Portuguese, with abstract in English).

Zhou, Q.; Liu, W.; Zhang, Y.; Liu, K.K. 2007. Action mechanisms of acetolactate synthase-inhibiting herbicides. Pesticide Biochemistry Physiology 89: 89-96. 